\title{
The genome sequence of the large burdock Cheilosia, Cheilosia
}

\section{vulpina (Meigen, 1822) [version 1; peer review: 2 approved]}

\author{
Steven Falk ${ }^{1}$, University of Oxford and Wytham Woods Genome Acquisition Lab, \\ Natural History Museum Genome Acquisition Lab, \\ Darwin Tree of Life Barcoding collective, \\ Wellcome Sanger Institute Tree of Life programme, \\ Wellcome Sanger Institute Scientific Operations: DNA Pipelines collective, \\ Tree of Life Core Informatics collective, Darwin Tree of Life Consortium \\ ${ }^{1}$ Independent Researcher, Kenilworth, UK
}

V1 First published: 17 Dec 2021, 6:351

https://doi.org/10.12688/wellcomeopenres.17491.1

Latest published: 17 Dec 2021, 6:351

https://doi.org/10.12688/wellcomeopenres.17491.1

\section{Abstract}

We present a genome assembly from an individual female Cheilosia vulpina (the large burdock Cheilosia or stocky blacklet; Arthropoda; Insecta; Diptera; Syriphidae). The genome sequence is 913 megabases in span. The majority of the assembly $(98.81 \%)$ is scaffolded into sixchromosomal pseudomolecules, with the $\mathrm{X}$ sex chromosome assembled.

\section{Keywords}

Cheilosia vulpina, large burdock Cheilosia, genome sequence, chromosomal, Diptera

\section{This article is included in the Tree of Life} gateway.

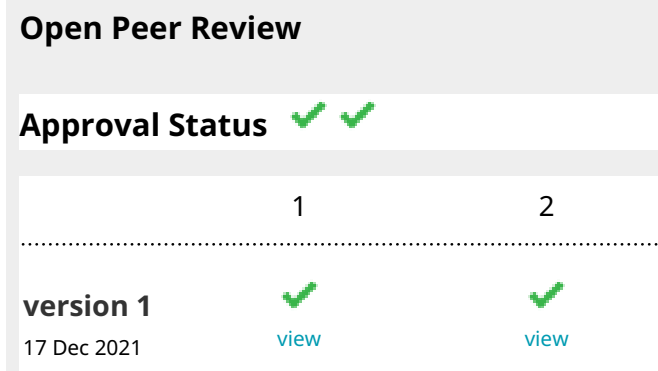

1. Nallur B. Ramachandra (D), University of Mysore, Mysuru, India

Koushik Ponnanna, University of Mysore, Mysore, India

2. Ljiljana Šašić Zorić ID), BioSense Institute, Novi Sad, Serbia

Any reports and responses or comments on the article can be found at the end of the article. 
Corresponding author: Darwin Tree of Life Consortium (mark.blaxter@sanger.ac.uk)

Author roles: Falk S: Investigation, Resources, Writing - Original Draft Preparation;

Competing interests: No competing interests were disclosed.

Grant information: This work was supported by Wellcome through core funding to the Wellcome Sanger Institute (206194) and the Darwin Tree of Life Discretionary Award (218328).

The funders had no role in study design, data collection and analysis, decision to publish, or preparation of the manuscript.

Copyright: (c) 2021 Falk S et al. This is an open access article distributed under the terms of the Creative Commons Attribution License, which permits unrestricted use, distribution, and reproduction in any medium, provided the original work is properly cited.

How to cite this article: Falk S, University of Oxford and Wytham Woods Genome Acquisition Lab, Natural History Museum Genome Acquisition Lab et al. The genome sequence of the large burdock Cheilosia, Cheilosia vulpina (Meigen, 1822) [version 1; peer review: 2 approved] Wellcome Open Research 2021, 6:351 https://doi.org/10.12688/wellcomeopenres.17491.1

First published: 17 Dec 2021, 6:351 https://doi.org/10.12688/wellcomeopenres.17491.1 


\section{Species taxonomy}

Eukaryota; Metazoa; Ecdysozoa; Arthropoda; Hexapoda; Insecta; Pterygota; Neoptera; Endopterygota; Diptera; Brachycera; Muscomorpha; Syrphoidea; Syrphidae; Eristalinae; Rhingiini; Cheilosia; Cheilosia vulpina (Meigen, 1822) (NCBI:txid273409).

\section{Background}

Cheilosia vulpina (the large burdock Cheilosia or the stocky blacklet) is a stocky, medium-large Cheilosia with outstanding hairs on the face. The males can be distinguished from other large hairy-faced Cheilosia by the longer body hairs, especially the scutellar marginals and hairs around the margins of the abdomen (much shorter in $C$. lasiopa) and pale bases to the tibiae (dark in the much longer-winged $C$. variabilis). The females have very noticeable 'fasciae' of pale hairs across the tergites and look like large, well-marked specimens of $C$. proxima or the scarcer $C$. velutina in the field (neither of which have outstanding facial hairs).

In the UK, $C$. vulpina is widespread though localised to southern England and seems to be most frequent in calcareous grasslands. It has been reared from the roots of greater and lesser burdocks in Germany (the probable foodplants in Britain) and also globe artichoke outside of the UK. There are two generations a year, and the spring brood averages larger and hairier than the summer one. The summer generation was once regarded as a separate species, $C$. conops. Adults are particularly keen on the flowers of umbellifers, including cow parsley in spring, and angelica, wild parsnip, and upright hedge-parsley in summer.

\section{Genome sequence report}

The genome was sequenced from a single female $C$. vulpina (Figure 1) collected from Wytham Woods, Oxfordshire (biological vice-county: Berkshire), UK (latitude 51.77, longitude -1.331). A total of 51-fold coverage in Pacific Biosciences single-molecule long reads and 120-fold coverage in $10 \mathrm{X}$

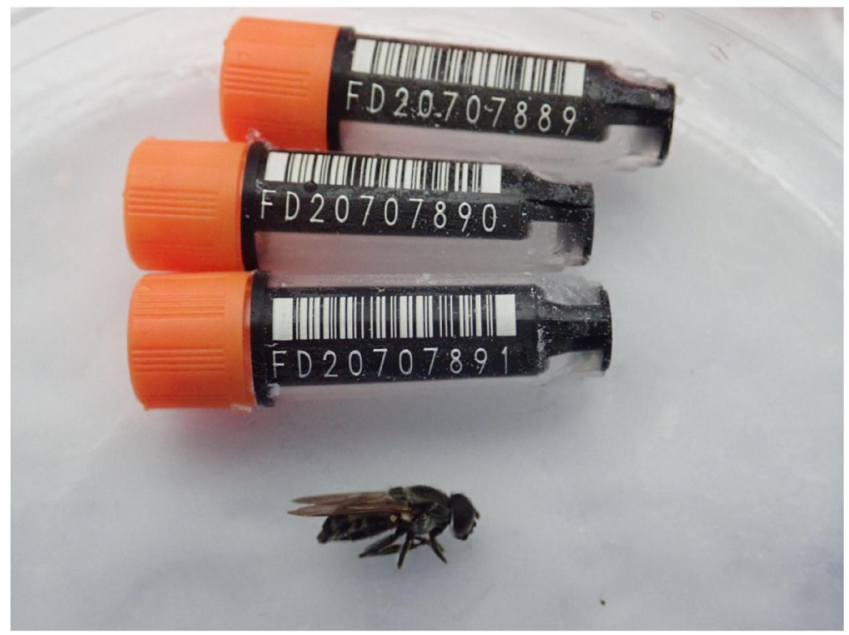

Figure 1. Image of the sequenced idCheVulp2 specimen taken during preservation and processing.
Genomics read clouds were generated. Primary assembly contigs were scaffolded with chromosome conformation Hi-C data. Manual assembly curation corrected $11 \mathrm{missing} / \mathrm{misjoins,} \mathrm{reduc-}$ ing the assembly length by $0.11 \%$ and the scaffold number by $33.33 \%$, and increasing the scaffold N50 by $52.44 \%$.

The final assembly has a total length of $405 \mathrm{Mb}$ in 20 sequence scaffolds with a scaffold N50 of $69.4 \mathrm{Mb}$ (Table 1). The majority, $99.92 \%$, of the assembly sequence was assigned to 6 chromosomal-level scaffolds, representing 5 autosomes (numbered by sequence length), and the X sex chromosome (Figure 2-Figure 5; Table 2). The assembly has a BUSCO completeness of $97.1 \%$ (single $96.7 \%$, duplicated $0.5 \%$ ) using the diptera_odb 10

Table 1. Genome data for Cheilosia vulpina, idCheVulp2.1.

\begin{tabular}{|c|c|}
\hline \multicolumn{2}{|l|}{ Project accession data } \\
\hline Assembly identifier & idCheVulp2.1 \\
\hline Species & Cheilosia vulpina \\
\hline Specimen & $\begin{array}{l}\text { idCheVulp2 (genome } \\
\text { assembly, Hi-C); idCheVulp1 } \\
\text { (Hi-C) }\end{array}$ \\
\hline NCBI taxonomy ID & 273409 \\
\hline BioProject & PRJEB46307 \\
\hline BioSample ID & SAMEA7746587 \\
\hline Isolate information & $\begin{array}{l}\text { Female, thorax, head } \\
\text { (idCheVulp2); Unknown sex, } \\
\text { head (idCheVulp1) }\end{array}$ \\
\hline \multicolumn{2}{|l|}{ Raw data accessions } \\
\hline PacificBiosciences SEQUEL II & ERR6807994 \\
\hline 10X Genomics Illumina & ERR6688460-ERR6688463 \\
\hline Hi-C Illumina & ERR6688459, ERR6688464 \\
\hline \multicolumn{2}{|l|}{ Genome assembly } \\
\hline Assembly accession & GCA_916610125.1 \\
\hline Accession of alternate haplotype & GCA_916610195.1 \\
\hline Span (Mb) & 405 \\
\hline Number of contigs & 30 \\
\hline Contig N50 length (Mb) & 45.5 \\
\hline Number of scaffolds & 20 \\
\hline Scaffold N50 length (Mb) & 69.4 \\
\hline Longest scaffold (Mb) & 117.5 \\
\hline BUSCO* genome score & $\begin{array}{l}\text { C:97.1\%[S:96.7\%,D:0.5\%], } \\
\text { F:0.7\%,M:2.1\%,n:3285 }\end{array}$ \\
\hline
\end{tabular}

*BUSCO scores based on the diptera odb10 BUSCO set using v5.1.2. $\mathrm{C}=$ complete $[\mathrm{S}=$ single copy, $\mathrm{D}=$ duplicated], $\mathrm{F}=$ fragmented, $\mathrm{M}=$ missing $\mathrm{n}=$ number of orthologues in comparison. A full set of BUSCO scores is available at https://blobtoolkit.genomehubs.org/view/idCheVulp2.1/dataset/ CAKAIU01/busco. 


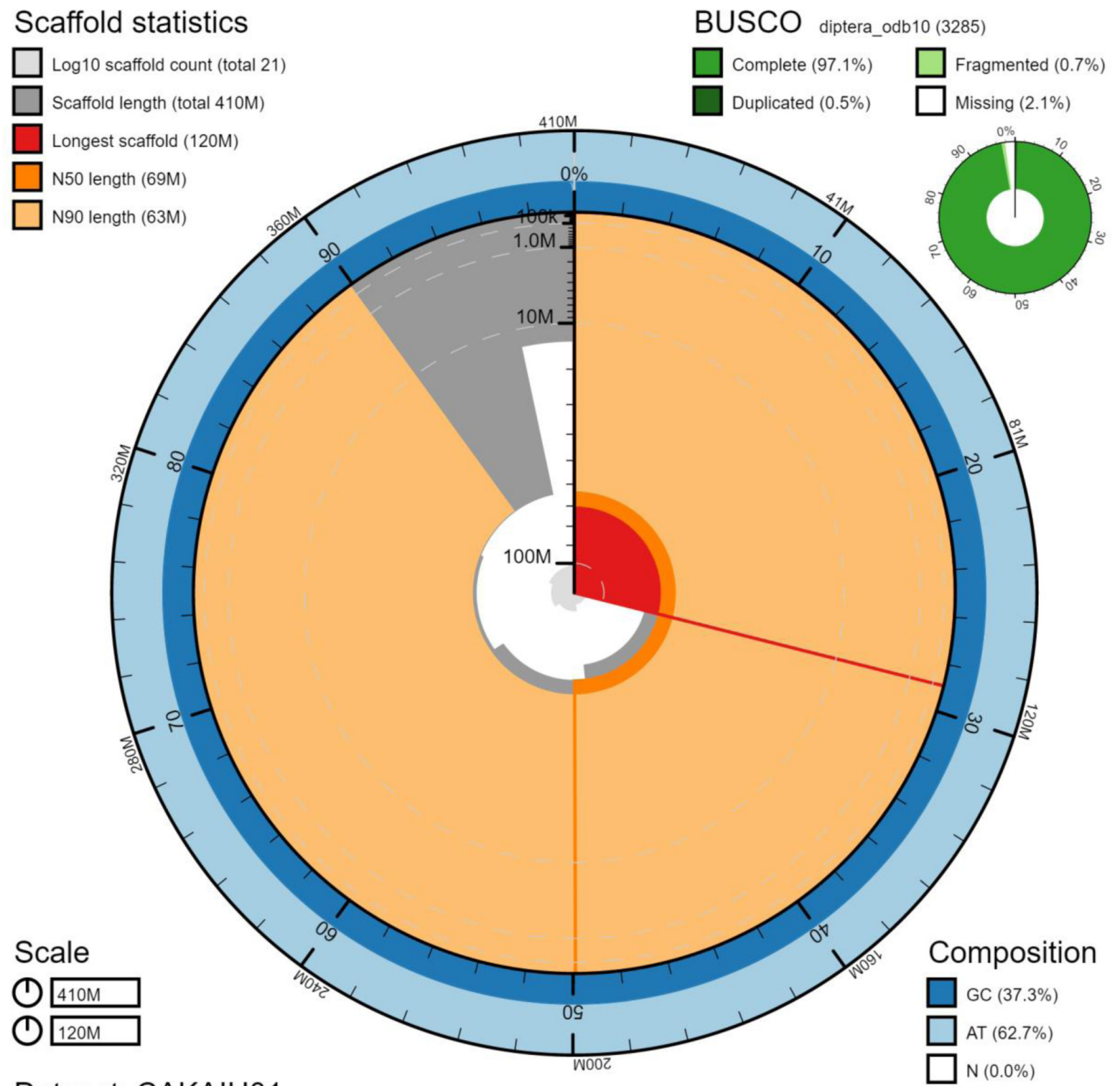

\section{Dataset: CAKAIU01}

Figure 2. Genome assembly of Cheilosia vulpina, idCheVulp2.1: metrics. The BlobToolKit Snailplot shows N50 metrics and BUSCO gene completeness. The main plot is divided into 1,000 size-ordered bins around the circumference with each bin representing $0.1 \%$ of the 405,383,207 bp assembly. The distribution of chromosome lengths is shown in dark grey with the plot radius scaled to the longest chromosome present in the assembly $(117,531,788 \mathrm{bp}$, shown in red). Orange and pale-orange arcs show the N50 and N90 chromosome lengths $(69,418,634$ and $63,252,922$ bp), respectively. The pale grey spiral shows the cumulative chromosome count on a log scale with white scale lines showing successive orders of magnitude. The blue and pale-blue area around the outside of the plot shows the distribution of GC, AT and N percentages in the same bins as the inner plot. A summary of complete, fragmented, duplicated and missing BUSCO genes in the diptera_odb10 set is shown in the top right. An interactive version of this figure is available at https://blobtoolkit.genomehubs.org/ view/idCheVulp2.1/dataset/CAKAIU01/snail.

reference set. While not fully phased, the assembly deposited is of one haplotype. Contigs corresponding to the second haplotype have also been deposited.

\section{Methods}

Sample acquisition and nucleic acid extraction

A female $C$. vulpina (idCheVulp2) and a second $C$. vulpina of unknown sex (idCheVulp1) were collected from Wytham
Woods, Oxfordshire (biological vice-county: Berkshire), UK (latitude 51.77, longitude -1.331) by Steven Falk, independent researcher, who also identified the specimens. The specimens were collected using a net and snap-frozen on dry ice.

DNA was extracted at the Tree of Life laboratory, Wellcome Sanger Institute. The idCheVulp2 sample was weighed and dissected on dry ice with tissue set aside for Hi-C sequencing. 


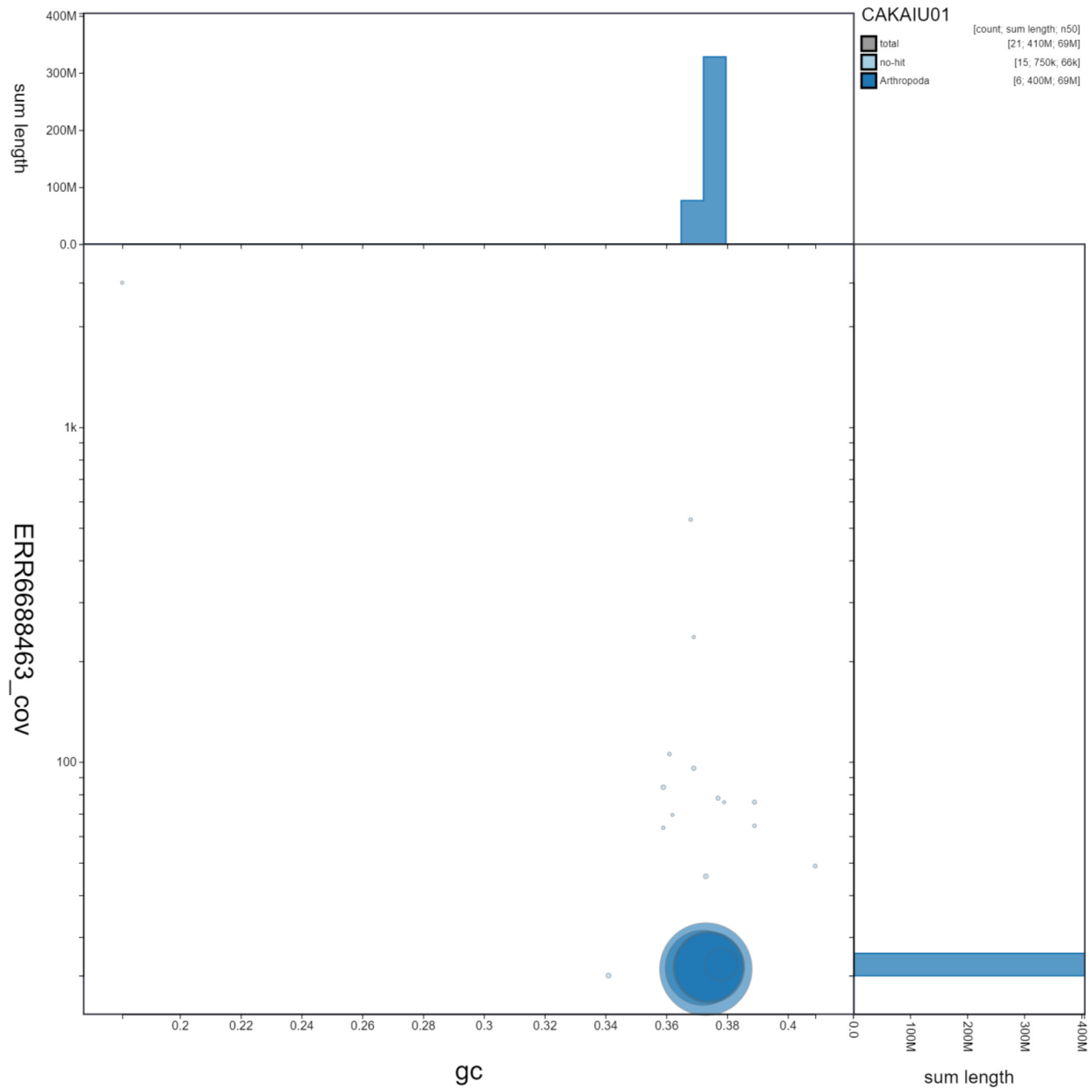

Figure 3. Genome assembly of Cheilosia vulpina, idCheVulp2.1: GC coverage. BlobToolKit GC-coverage plot. Scaffolds are coloured by phylum. Circles are sized in proportion to scaffold length. Histograms show the distribution of scaffold length sum along each axis. An interactive version of this figure is available at https://blobtoolkit.genomehubs.org/view/idCheVulp2.1/dataset/CAKAIU01/blob.

Abdomen tissue was disrupted using a Nippi Powermasher fitted with a BioMasher pestle. Fragment size analysis of $0.01-0.5 \mathrm{ng}$ of DNA was then performed using an Agilent FemtoPulse. High molecular weight (HMW) DNA was extracted using the Qiagen MagAttract HMW DNA extraction kit. Low molecular weight DNA was removed from a 200-ng aliquot of extracted DNA using $0.8 \mathrm{X}$ AMpure $\mathrm{XP}$ purification kit prior to $10 \mathrm{X}$
Chromium sequencing; a minimum of 50 ng DNA was submitted for $10 \mathrm{X}$ sequencing. HMW DNA was sheared into an average fragment size between $12-20 \mathrm{~kb}$ in a Megaruptor 3 system with speed setting 30. Sheared DNA was purified by solidphase reversible immobilisation using AMPure PB beads with a $1.8 \mathrm{X}$ ratio of beads to sample to remove the shorter fragments and concentrate the DNA sample. The concentration of the sheared 


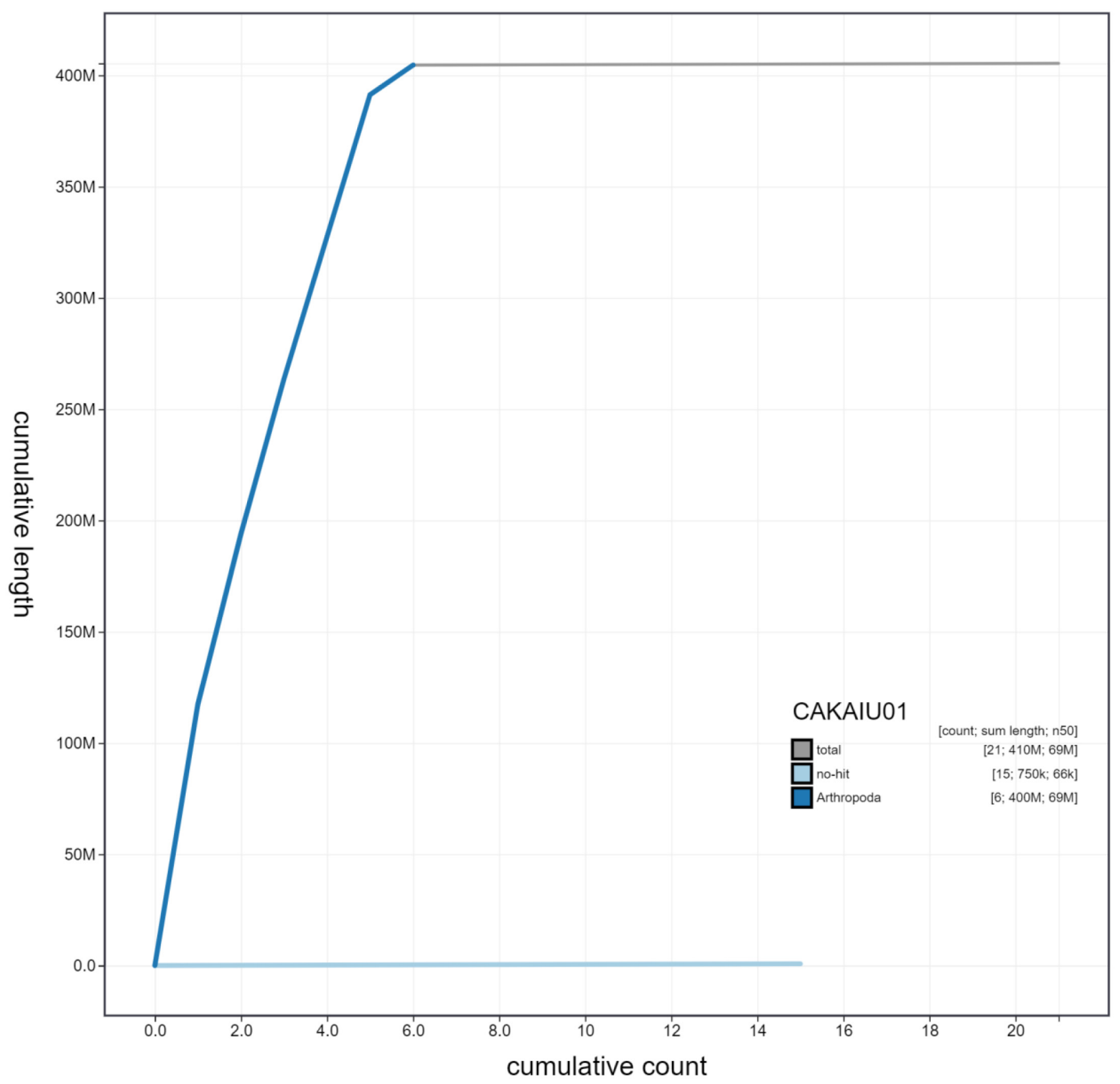

Figure 4. Genome assembly of Cheilosia vulpina, idCheVulp2.1: cumulative sequence. BlobToolKit cumulative sequence plot. The grey line shows cumulative length for all scaffolds. Coloured lines show cumulative lengths of scaffolds assigned to each phylum using the buscogenes taxrule. An interactive version of this figure is available at https://blobtoolkit.genomehubs.org/view/idCheVulp2.1/dataset/ CAKAIU01/cumulative.

and purified DNA was assessed using a Nanodrop spectrophotometer and Qubit Fluorometer and Qubit dsDNA High Sensitivity Assay kit. Fragment size distribution was evaluated by running the sample on the FemtoPulse system.

\section{Sequencing}

Pacific Biosciences HiFi circular consensus and 10X Genomics Chromium read cloud sequencing libraries were constructed according to the manufacturers' instructions. Sequencing was performed by the Scientific Operations core at the Wellcome Sanger Institute on Pacific Biosciences SEQUEL II and Illumina NovaSeq 6000 instruments. Hi-C data were generated from head tissue of idChrBicil and idCheVulp2 using the Arima Hi-C+ kit and sequenced on a NovaSeq 6000 instrument.

\section{Genome assembly}

Assembly was carried out with Hifiasm (Cheng et al., 2021); haplotypic duplication was identified and removed with 


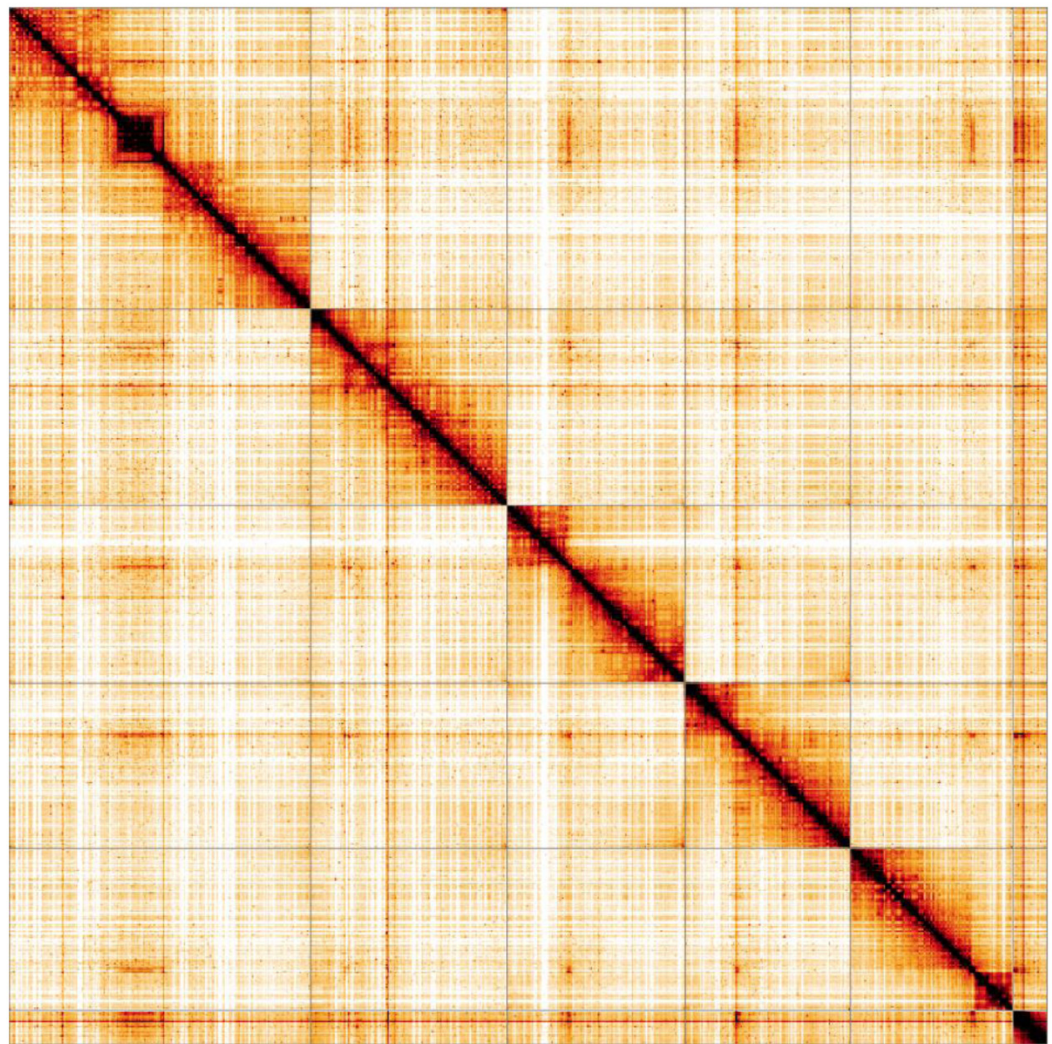

Figure 5. Genome assembly of Cheilosia vulpina, idCheVulp2.1: Hi-C contact map. Hi-C contact map of the icCheVulp2.1 assembly, visualised in HiGlass. Chromosomes are given in order of size from left to right and top to bottom.

Table 2. Chromosomal pseudomolecules in the genome assembly of Cheilosia vulpina, idCheVulp2.1.

\begin{tabular}{|c|c|c|c|}
\hline INSDC accession & Chromosome & Size $\mathbf{( M b )}$ & GC\% \\
\hline OU744276.1 & 1 & 117.53 & 37.3 \\
\hline OU744277.1 & 2 & 76.59 & 37.2 \\
\hline OU744278.1 & 3 & 69.42 & 37.4 \\
\hline OU744279.1 & 4 & 64.49 & 37.4 \\
\hline OU744280.1 & 5 & 63.25 & 37.4 \\
\hline OU744281.1 & $X$ & 13.34 & 37.8 \\
\hline OU744282.1 & MT & 0.02 & 18.1 \\
\hline- & Unplaced & 0.74 & 36.9 \\
\hline
\end{tabular}

purge_dups (Guan et al., 2020). One round of polishing was performed by aligning $10 \mathrm{X}$ Genomics read data to the assembly with longranger align, calling variants with freebayes (Garrison \& Marth, 2012). The assembly was then scaffolded with Hi-C data (Rao et al., 2014) using SALSA2 (Ghurye et al., 2019). The assembly was checked for contamination and corrected using the gEVAL system (Chow et al., 2016) as described previously (Howe et al., 2021). Manual curation was performed using gEVAL, HiGlass (Kerpedjiev et al., 2018) and Pretext. The mitochondrial genome was assembled using MitoHiFi (Uliano-Silva et al., 2021), with annotation performed using MitoFinder. The genome was analysed and BUSCO scores generated within the BlobToolKit environment (Challis et al., 2020). Table 3 contains a list of all software tool versions used, where appropriate.

\section{Ethics/compliance issues}

The materials that have contributed to this genome note have been supplied by a Darwin Tree of Life Partner. The submission of materials by a Darwin Tree of Life Partner is subject to the Darwin Tree of Life Project Sampling Code of Practice. By agreeing with and signing up to the Sampling Code of Practice, the Darwin Tree of Life Partner agrees they will meet the legal and ethical requirements and standards set out within this document in respect of all samples acquired for, and supplied to, the Darwin Tree of Life Project. Each transfer of samples is further undertaken according to a Research Collaboration Agreement or Material Transfer Agreement entered into by the Darwin Tree of Life Partner, Genome Research Limited (operating as the Wellcome Sanger Institute), and in some circumstances other Darwin Tree of Life collaborators. 
Table 3. Software tools used.

\begin{tabular}{|l|l|l|}
\hline Software tool & Version & Source \\
\hline Hifiasm & $0.15 .3-$ r339 & Cheng et al., 2021 \\
\hline purge_dups & 1.2 .3 & Guan et al., 2020 \\
\hline SALSA2 & 2.2 & Ghurye et al., 2019 \\
\hline longranger align & 2.2 .2 & $\begin{array}{l}\text { https://support.10xgenomics.com/genome-exome/ } \\
\text { software/pipelines/latest/advanced/other-pipelines }\end{array}$ \\
\hline freebayes & $1.3 .1-17-g a a 2 a c e 8$ & Garrison \& Marth, 2012 \\
\hline MitoHiFi & 2.0 & Uliano-Silva et al., 2021 \\
\hline HiGlass & 1.11 .6 & Kerpedjiev et al., 2018 \\
\hline PretextView & $0.2 . x$ & https://github.com/wtsi-hpag/PretextView \\
\hline BlobToolKit & 2.6 .2 & Challis et al., 2020 \\
\hline
\end{tabular}

\section{Data availability}

European Nucleotide Archive: Cheilosia vulpina (large burdock Cheilosia). Accession number PRJEB46307: https://www.ebi. ac.uk/ena/browser/view/PRJEB46307.

The genome sequence is released openly for reuse. The C. vulpina genome sequencing initiative is part of the Darwin Tree of Life (DToL) project. All raw sequence data and the assembly have been deposited in INSDC databases. The genome will be annotated and presented through the Ensembl pipeline at the European Bioinformatics Institute. Raw data and assembly accession identifiers are reported in Table 1.

\section{Author information}

Members of the University of Oxford and Wytham Woods Genome Acquisition Lab are listed here: https://doi.org/10.5281/ zenodo. 5746938 .
Members of the Darwin Tree of Life Barcoding collective are listed here: https://doi.org/10.5281/zenodo.5744972.

Members of the Wellcome Sanger Institute Tree of Life programme are listed here: https://doi.org/10.5281/zenodo. 5744840 .

Members of Wellcome Sanger Institute Scientific Operations: DNA Pipelines collective are listed here: https://doi.org/10.5281/ zenodo. 5746904.

Members of the Tree of Life Core Informatics collective are listed here: https://doi.org/10.5281/zenodo.5743293.

Members of the Darwin Tree of Life Consortium are listed here: https://doi.org/10.5281/zenodo.5638618.

\section{Acknowledgements}

The Background section has been adapted from Steven Falk's flickr album.
Challis R, Richards E, Rajan J, et al.: BlobToolKit - Interactive Quality Assessment of Genome Assemblies. G3 (Bethesda). 2020; 10(4): 1361-74. PubMed Abstract | Publisher Full Text | Free Full Text

Cheng $\mathrm{H}$, Concepcion GT, Feng X, et al.: Haplotype-Resolved de Novo Assembly Using Phased Assembly Graphs with Hifiasm. Nat Methods. 2021; 18(2): 170-75. PubMed Abstract | Publisher Full Text | Free Full Text

Chow W, Brugger K, Caccamo M, et al.: gEVAL - a web-based browser for evaluating genome assemblies. Bioinformatics. 2016; 32(16): 2508-10. PubMed Abstract | Publisher Full Text | Free Full Text

Garrison E, Marth G: Haplotype-Based Variant Detection from Short-Read
Sequencing. 2012; arXiv: 1207.3907.

Reference Source

Ghurye J, Rhie A, Walenz BP, et al.: Integrating Hi-C Links with Assembly Graphs for Chromosome-Scale Assembly. PLoS Comput Biol. 2019; 15(8): e1007273.

PubMed Abstract | Publisher Full Text | Free Full Text

Guan D, McCarthy SA, Wood J, et al.: Identifying and Removing Haplotypic Duplication in Primary Genome Assemblies. Bioinformatics. 2020; 36(9): 2896-98.

PubMed Abstract | Publisher Full Text | Free Full Text 
Howe K, Chow W, Collins J, et al:: Significantly Improving the Quality of Genome Assemblies through Curation. GigaScience. 2021; 10(1): giaa153. PubMed Abstract | Publisher Full Text | Free Full Text

Kerpedjiev P, Abdennur N, Lekschas F, et al.: HiGlass: Web-Based Visual Exploration and Analysis of Genome Interaction Maps. Genome Biol. 2018, 1(1): 125 .

PubMed Abstract | Publisher Full Text | Free Full Text
Rao SSP, Huntley MH, Durand NC, et al:: A 3D Map of the Human Genome at Kilobase Resolution Reveals Principles of Chromatin Looping. Cell. 2014; 159(7): 1665-80.

PubMed Abstract | Publisher Full Text | Free Full Text

Uliano-Silva M, Nunes JGF, Krasheninnikova K, et al.: marcelauliano/MitoHiFi: mitohifi_v2.0. 2021.

Publisher Full Text 


\section{Open Peer Review}

\section{Current Peer Review Status:}

\section{Version 1}

Reviewer Report 11 November 2022

https://doi.org/10.21956/wellcomeopenres.19341.r53053

(C) 2022 Zorić L. This is an open access peer review report distributed under the terms of the Creative Commons Attribution License, which permits unrestricted use, distribution, and reproduction in any medium, provided the original work is properly cited.

\section{Ljiljana Šašić Zorić}

BioSense Institute, Novi Sad, Serbia

"The genome sequence of the large burdock Cheilosia, Cheilosia vulpina (Meigen, 1822)" is presented in the form of a technical report on the genome assembly of hoverfly species Cheilosia vulpina. The article is an important contribution to further research on hoverflies being known as the second most important pollinators among insects. It describes technical aspects of genome assembly clearly and concisely. The applied approach includes PacBio and 10X sequencing data, with scaffolding using $\mathrm{Hi}-\mathrm{C}$ data. The methodology is suitable and overall well described, although some minor inconsistencies should be resolved before final publication.

Suggestions for revision:

Abstract

- The genome assembly total length and per cent of the assembly sequence assigned to 6 chromosomal-level scaffolds (913M and $98.81 \%$ ) are not in agreement with the main text (405M and 99.92\%).

"sixchromosomal" should be written separately "six chromosomal"

Genome sequence report

It would be good if the caption for Figure 1 refers to the name of the species, not only ID. I would suggest editing the figure caption in the following way: "Image of the sequenced Cheilosia vulpina (idCheVulp2) specimen..."

In the second paragraph, it is stated that the final assembly has a total length of $405 \mathrm{Mb}$ in 20 sequence scaffolds. The same is stated in Table 1. However, based on Figure 2 and results available at https://blobtoolkit.genomehubs.org/view/idCheVulp2.1/dataset/CAKAIU01/snail it seems that there are 21 scaffolds.

Figure 2 is a bit different compared to the interactive version on the link https://blobtoolkit.genomehubs.org/view/idCheVulp2.1/dataset/CAKAIU01/snail (scale in 
the interactive version is set to 405 and $118 \mathrm{M}$, while in the text is $410 \mathrm{M}$ and $120 \mathrm{M}$ ). Please check this.

Figure 3 needs some technical corrections such as first letter capitalization in the names of axes and gc should also be capitalized

Methods

It is stated that the gender of specimen idCheVulp1 is not known. What is the reason not to determine gender? Is the specimen damaged? Please state this.

There is inconsistency in the text regarding the use of idCheVulp1 specimen in analysis. Although based on Table 1 it is used for $\mathrm{Hi}-\mathrm{C}$, only for idCheVulp2 sample is stated that tissue was set aside for $\mathrm{Hi}-\mathrm{C}$ sequencing. Additionally, it is written that $\mathrm{Hi}-\mathrm{C}$ data were generated from the head tissue of idChrBici1 and idCheVulp2, but there is not any additional information for specimen idChrBici1.

There is no information on parameters used in data analysis. If the default values were used please state that clearly or, in case the data analysis follows the procedure previously described in detail, please refer to the source.

Is the rationale for creating the dataset(s) clearly described?

Yes

Are the protocols appropriate and is the work technically sound?

Yes

Are sufficient details of methods and materials provided to allow replication by others? Partly

Are the datasets clearly presented in a useable and accessible format?

Yes

Competing Interests: No competing interests were disclosed.

Reviewer Expertise: Population genetics and molecular taxonomy

I confirm that I have read this submission and believe that I have an appropriate level of expertise to confirm that it is of an acceptable scientific standard.

Reviewer Report 18 January 2022

https://doi.org/10.21956/wellcomeopenres.19341.r47741

(C) 2022 Ramachandra $\mathbf{N}$ et al. This is an open access peer review report distributed under the terms of the Creative Commons Attribution License, which permits unrestricted use, distribution, and reproduction in any medium, provided the original work is properly cited. 


\section{Nallur B. Ramachandra}

Department of Studies in Genetics and Genomics, University of Mysore, Mysuru, Karnataka, India Koushik Ponnanna

University of Mysore, Mysore, India

Genome sequencing of plants and animals, including humans, is generating enormous data to understand the biology, structure, and function of the organism. One such event is this

manuscript wherein a single female of the large burdock Cheilosia vulpina (Meigen, 1822) is being reported.

The methodology followed for DNA isolation, sequencing, and assembling the genome is well organized. The organelle DNA is also taken into account with necessary contamination removal. This kind of information certainly adds to the genome resources of insects.

However, we encountered the following concerns:

1. Throughout the manuscript, it is mentioned that a single female is sequenced. Is it impregnated or virgin? If it is impregnated, how do you account for possible male-specific genomic content?

2. Information about the available genome studies about $C$. vulpina is lacking in this manuscript.

3. Comparison of this assembly with the already available genome would have helped in appreciating the accuracy achieved.

4. Long read sequencing generally introduces sequencing errors. How was this taken care of in this study?

Overall, the data will definitely add to the existing genomic resources for $C$. vulpina which otherwise lacks a rich resource necessary for comparative studies.

Is the rationale for creating the dataset(s) clearly described?

Yes

Are the protocols appropriate and is the work technically sound?

Yes

Are sufficient details of methods and materials provided to allow replication by others? Partly

Are the datasets clearly presented in a useable and accessible format?

Yes

Competing Interests: No competing interests were disclosed.

Reviewer Expertise: Genomics of Drosophila and Humans

We confirm that we have read this submission and believe that we have an appropriate level 
of expertise to confirm that it is of an acceptable scientific standard. 